\title{
JULGAMENTO AUTOMATIZADO DA QUALIDADE SUPERFICIAL DE BOBINAS LAMINADAS A QUENTE NA ARCELORMITTAL TUBARÃO*
}

\author{
Leonardo Zuchi Guio' \\ Leandro de Lucas Mendes² \\ Roberto Dalmaso ${ }^{3}$ \\ Amarildo Gomes Dutra
}

\section{Resumo}

A ArcelorMittal Tubarão vem trabalhando nos últimos dois anos na implantação de um sistema automatizado de julgamento da qualidade da superfície de bobinas laminadas a quente, juntamente com a ArcelorMittal Global R\&D. Tal sistema é denominado CGSIS - Coil Grading Surface Inspection System - e tem a função de interpretar as ocorrências de defeitos superficiais, identificados por um sistema de inspeção automático através de câmeras CCD auxiliado por técnicas de visão artificial, à luz da necessidade requerida pela aplicação final do material. Todas as ações sugeridas pelo sistema são baseadas em regras de aplicação e nas informações obtidas do sistema de inspeção automático, requerendo para isso taxas de acerto de classificação de defeitos adequadas para uma boa tomada de decisão. Para tanto, várias melhorias foram necessárias, envolvendo questões de ambiente, desempenho, confiabilidade, disponibilidade e treinamento do sistema, para se obter com isso melhores níveis de detecção e acerto. Outras questões envolvidas em tal processo concernem às mudanças aplicadas a políticas já estabelecidas de julgamento e comunicação do novo sistema com os sistemas já existentes. Vários benefícios da implantação do projeto já estão sendo percebidos provenientes das etapas de preparação para a instalação e espera-se que após a operacionalização um ganho superior a $R \$ 320 \mathrm{k}$ entre redução de custo com pessoal, aproveitamento de material e redução de reclamações de clientes seja alcançado.

Palavras-chave: Inspeção; Qualidade; Bobina; Laminação.

\section{AUTOMATED HOT ROLLED COIL SURFACE QUALITY JUDGEMENT AT ARCELORMITTAL TUBARAO}

\section{Abstract}

ArcelorMittal Tubarao has worked in the last two years with ArcelorMittal Global R\&D on the installation of an automated hot rolled coil surface quality judgement system. This system is called CGSIS - Coil Grading Surface Inspection System - and has the function to interpret superficial defects occurrences, identified by an automated inspection system which uses CCD cameras and artificial vision techniques, regarding the quality required for the product application. All the actions suggested by the system are based in application rules and information from the automated inspection system, requiring high defects classification rates. For this, some improvements were done, involving environment issues, performance, reliability, availability and system training, to achieve better confidence and detection rates. Other issues involved in this process concern to the changes made to the existing judgement policies and communications between the new system and the existing systems. Many project implementation benefits are being noticed from preparation steps and it is expected a profit upper than $R \$ 320 \mathrm{k}$ regarding personnel reduction, product reuse and customer complaint reduction with system running.

Keywords: Inspection; Quality; Hot Rolled Coil; Rolling.

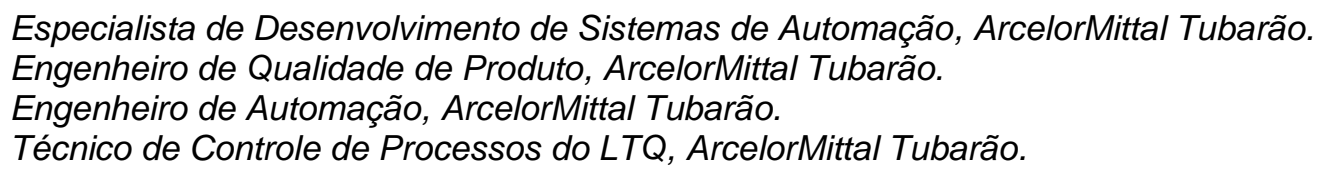

\footnotetext{
* Contribuição técnica ao $18^{\circ}$ Seminário de Automação e TI Industrial, 23 a 26 de setembro de 2014,
} São Paulo, SP, Brasil. 


\section{INTRODUÇÃO}

O julgamento da qualidade da superfície de bobinas laminadas a quente é tão crítico para a garantia da qualidade do produto quanto o julgamento da qualidade mecânica/dimensional. Tal processo consiste em verificar se o produto atende aos critérios de qualidade superficial definidos para a aplicação a que ele se destina. A verificação da qualidade superficial leva em consideração os defeitos encontrados e classificados na bobina durante o processo de inspeção do produto.

O julgamento da qualidade da superfície pode ser feito de forma automatizada ou não. Quando não é feito de forma automatizada, o critério de julgamento pode não ser bem definido e variar de acordo com o responsável pelo julgamento, bem como pode não levar em conta a totalidade dos defeitos encontrados na bobina e suas características.

O sistema de julgamento automático de bobinas é um sistema que recebe as informações do processo de inspeção da bobina e, considerando regras bem definidas de julgamento e a aplicação do produto, determina a qualidade superficial da bobina produzida, liberando-a para o cliente caso os critérios de qualidade sejam atendidos.

\section{A IMPLANTAÇÂO DE UM SISTEMA DE JULGAMENTO AUTOMATIZADO DE QUALIDADE SUPERFICIAL DE BOBINAS}

O CGSIS - Coil Grading Surface Inspection System - é um sistema desenvolvido pelo Centro de Pesquisa da ArcelorMittal Europa que permite o julgamento automático da qualidade das bobinas com base nos defeitos recebidos de um sistema de inspeção automática. Seu objetivo é dar suporte ao processo de tomada de decisão ao avaliar um mapa de defeitos superficiais de cada bobina produzida em confronto com requisitos de qualidade pré-definidos para a aplicação a que a bobina se destina.

O CGSIS já é utilizado há mais de 14 anos em linhas de galvanização, no entanto para linhas de tiras a quente seu desenvolvimento é recente. Isto se deve à complexidade das condições de processo, onde temos interferência de vapor, água, temperatura, entre outros.

A ArcelorMittal Tubarão vem há dois anos realizando modificações visando a melhoria do processo de inspeção automática e mais recentemente ajustando o sistema para a implantação do sistema CGSIS, de forma a garantir que todos os requisitos para implantação do sistema, tanto no que concerne à confiabilidade dos dados de defeitos que serão enviados ao CGSIS quanto nos requisitos técnicos de hardware, software e integração com outros sistemas.

A redução de esforços para julgar a qualidade superficial das bobinas é o maior objetivo do projeto, resultando em menor necessidade de recursos para o processo de julgamento. A uniformização do resultado de julgamento devido a um processo automatizado com base em critérios pré-estabelecidos é também um objetivo valoroso do projeto.

Outros ganhos podem são previstos, como a redução de tempo de julgamento, a possibilidade de acompanhamento online do desempenho e confiança do sistema de inspeção e simulação de corte para aproveitamento de bobinas.

\footnotetext{
* Contribuição técnica ao $18^{\circ}$ Seminário de Automação e TI Industrial, 23 a 26 de setembro de 2014 ,
} São Paulo, SP, Brasil. 


\subsection{Melhorias no Sistema de Inspeção Superficial}

O sistema de inspeção superficial da ArcelorMittal Tubarão, fornecido pela empresa alemã Parsytec, utilizava câmeras CCD analógicas, computadores industriais e unidades de iluminação alógenas. Tal arquitetura apresentava fragilidades: obsolescência dos componentes, alto consumo de bulbos alógenos, imagens distorcidas e com iluminação deficientes e alto consumo e baixo desempenho dos componentes. Para resolver as fragilidades, várias alterações foram feitas no sistema: atualização tecnológica dos computadores, movimentação dos computadores para as salas de inspeção eliminando componentes, instalação de câmeras digitais para captura das imagens e troca da iluminação alógena por lâmpadas a LED, com durabilidade maior e melhor controle da iluminação. Essas modificações contribuíram significativamente para a redução de consumo de componentes, aumento da disponibilidade de sobressalentes e aumento da qualidade das imagens de defeitos, contribuindo para maior confiabilidade dos resultados do processo de inspeção, resultados que são a principal informação requerida pelo CGSIS.

Já visando a instalação do sistema CGSIS, foram necessárias adequações ao sistema de inspeção especificamente nos aspectos de qualidade da detecção dos defeitos e do julgamento, para que fosse atingido um nível de confiança adequado nos seus resultados. Com o sistema Parsytec capturando imagens com mais qualidade, foi necessária a criação de novos classificadores de defeitos, de forma que o sistema fosse treinado a julgar os defeitos com as novas imagens.

Também na perspectiva de aumento da confiabilidade dos resultados de inspeção, ocorrências que comprometiam o desempenho do sistema tiveram que ser trabalhadas. Uma das ocorrências que mais impactavam no desempenho do sistema Parsytec era a detecção de imagens de água. A presença de água na superfície da tira era detectada pelo sistema e quando em grande volume resultava em descarte de imagens, as quais poderiam conter algum defeito relevante. Em alguns dias eram capturadas mais de 2000 imagens de água por bobina. Ajustes foram realizados na linha de processo com ajuste dos "cross sprays" e reposicionamento dos ventiladores na saída do trem acabador, com consequente redução do número de imagens para níveis abaixo de 100 imagens por bobina. Outra ocorrência que precisava ser reduzida era a de detecção de rolo. Em algumas condições desfavoráveis de iluminação, o sistema de inspeção apresentava dificuldades em determinar a borda do material, implicando na captura de imagens dos rolos da mesa como se fossem defeitos da tira. Além de uma melhoria na regularidade de limpeza das lentes, foi necessário alterar configurações do sistema no algoritmo de detecção de borda, melhorando sua precisão ao eliminar do processamento a área de brilho do rolo. Com as modificações houve uma redução significativa da quantidade de imagens de rolo, reduzindo de 1400 imagens em alguns casos para níveis próximos de zero.

Com essas melhorias, o acerto de classificação subiu de $21 \%$ para cerca de $80 \%$ até o momento. Até então o acerto de classificação não era crítico para o controle de qualidade, pois todas as imagens no sistema de inspeção automática eram avaliadas pelos inspetores que corrigiam as anormalidades quando necessário, porém, para o CGSIS julgar automaticamente, o acerto da classificação é prérequisito para implantação do CGSIS.

* Contribuição técnica ao $18^{\circ}$ Seminário de Automação e TI Industrial, 23 a 26 de setembro de 2014, São Paulo, SP, Brasil. 


\subsection{Preparação da Infraestrutura}

O CGSIS usa uma estrutura redundante para garantir alta disponibilidade e redução de risco de perda de dados. Existem 4 instâncias do sistema (Figura 1): CGSIS Prod, também chamada de Inspector, executa online e é usada para a tomada de decisão quanto ao julgamento da bobina, CGSIS Spare, instância redundante do CGSIS Prod, CGSIS Test, usada para validar modificações no sistema antes de aplica-las à produção, e CGSIS Expert, instância usada pelo especialista de qualidade para geração de novas configurações. As instâncias foram distribuídas em 2 servidores com sistema operacional Windows 2008 Server com 8GB de memória RAM e 700GB de disco rígido.
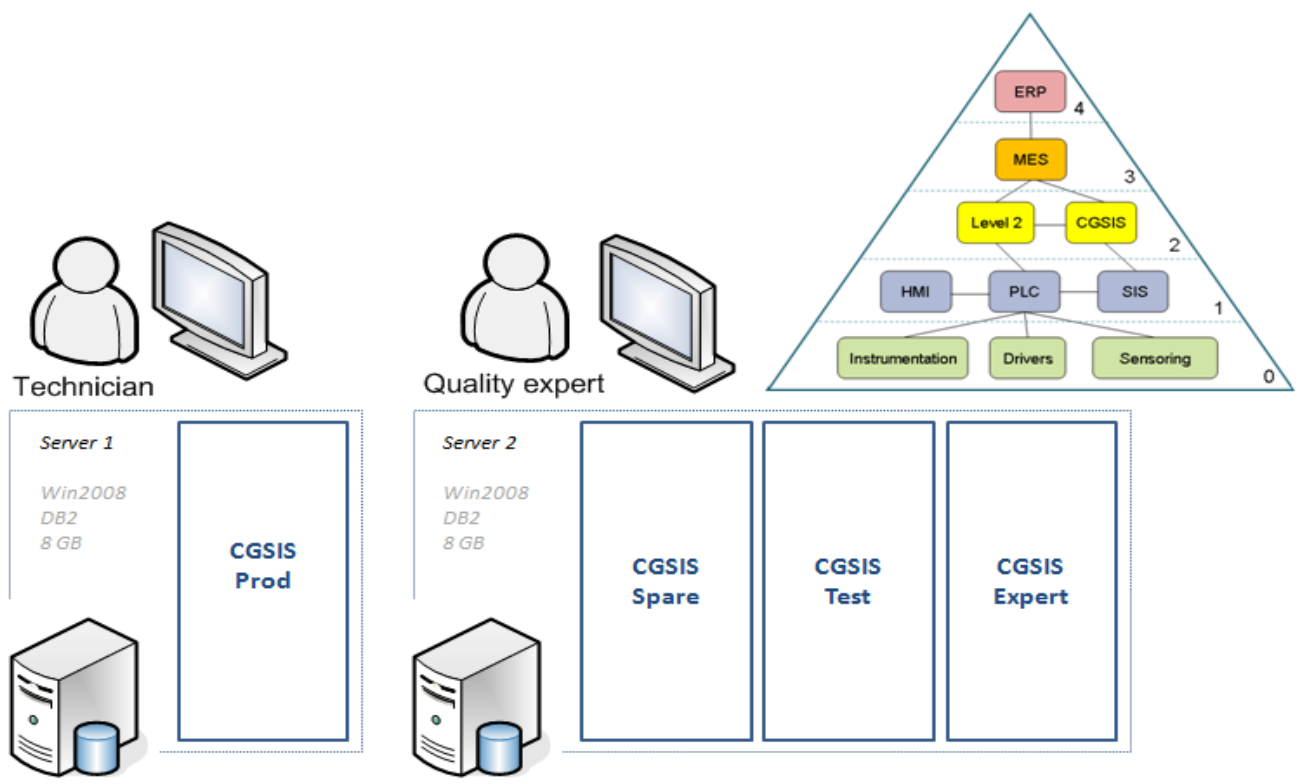

Figura 1. Estrutura do CGSIS e suas instâncias.

Outro sistema desenvolvido pelo $R \& D$, chamado EPC, já se encontrava implantado e em uso em Tubarão. Devido a esse sistema usar o mesmo framework do CGSIS e arquitetura semelhante, optou-se por instalar o CGSIS nessa mesma estrutura, porém, isso demandou um grande trabalho de preparação dos servidores. O EPC também possui dois servidores, sendo que um deles era compartilhado com um sistema de desenvolvimento com limitação de uso em sistema operacional 32 bits chamado LMS. Como o CGSIS requer sistema operacional 64 bits, optou-se por migrar o EPC utilizando mais um servidor. Foi feito um planejamento detalhado de migração do EPC com atuações em paradas de planta para garantir que não houvesse indisponibilidade do EPC (Figura 2).

* Contribuição técnica ao $18^{\circ}$ Seminário de Automação e TI Industrial, 23 a 26 de setembro de 2014, São Paulo, SP, Brasil. 


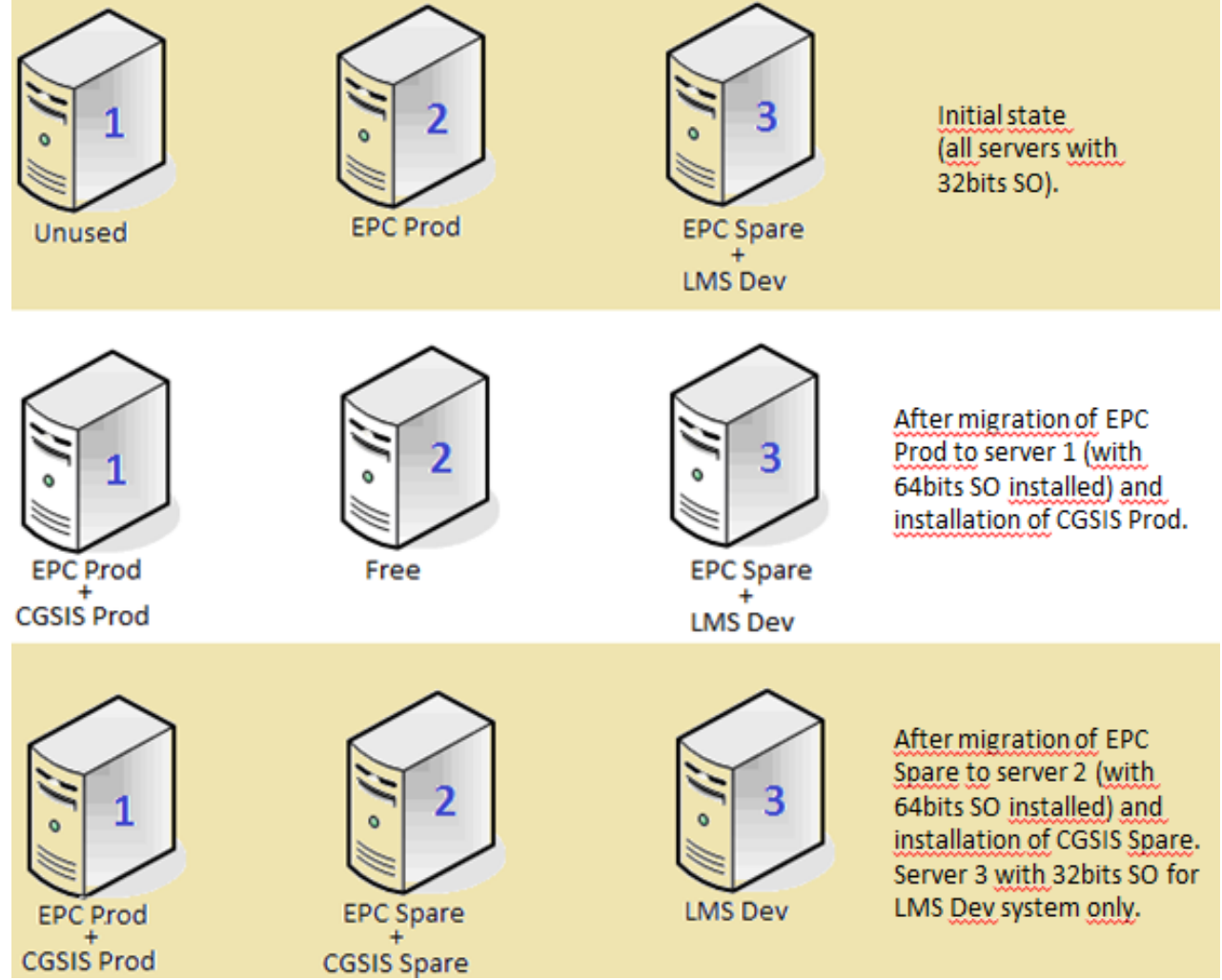

Figura 2. Etapas de migração do EPC e instalação do CGSIS.

Inicialmente o servidor 1 foi preparado e durante uma parada foi feita a migração do EPC Prod para esse servidor, logo em seguida tendo sido instalado o CGSIS Prod. A arquitetura redundante do EPC, garantia que caso algum problema fosse detectado no EPC Prod já migrado, a contingência não migrada que estava no servidor 3 poderia ser acionada até que o problema fosse solucionado. Durante um período o sistema EPC Prod migrado foi acompanhado pelo especialista para garantir seu funcionamento adequado. Nesse período o servidor 2 foi preparado para receber o EPC Test+Spare e o CGSIS Test+Spare+Expert. Após a validação da migração para o servidor 1 foram instalados no servidor 2 o EPC Test+Spare e o CGSIS Test+Spare+Expert, liberando o servidor 3 para uso apenas pelo sistema LMS Dev, que é limitado a sistema operacional 32 bits.

\subsection{Desenvolvimento das Interfaces de Comunicação}

O CGSIS se comunica com o Parsytec para recebimento do resultado da deteç̧ão e classificação dos defeitos e com o sistema de nível 2 do LTQ para receber informações de processo da bobina (Figura 3). Ambas as comunicações são realizadas via FTP e tanto os processos de comunicação como os arquivos com os dados tiveram que ser desenvolvidos nesses sistemas para prover ao CGSIS as informações necessárias. A comunicação com o sistema de execução da produção (MES) não é requisito para implantação do CGSIS e será desenvolvida em etapa futura do projeto.

* Contribuição técnica ao $18^{\circ}$ Seminário de Automação e TI Industrial, 23 a 26 de setembro de 2014, São Paulo, SP, Brasil. 


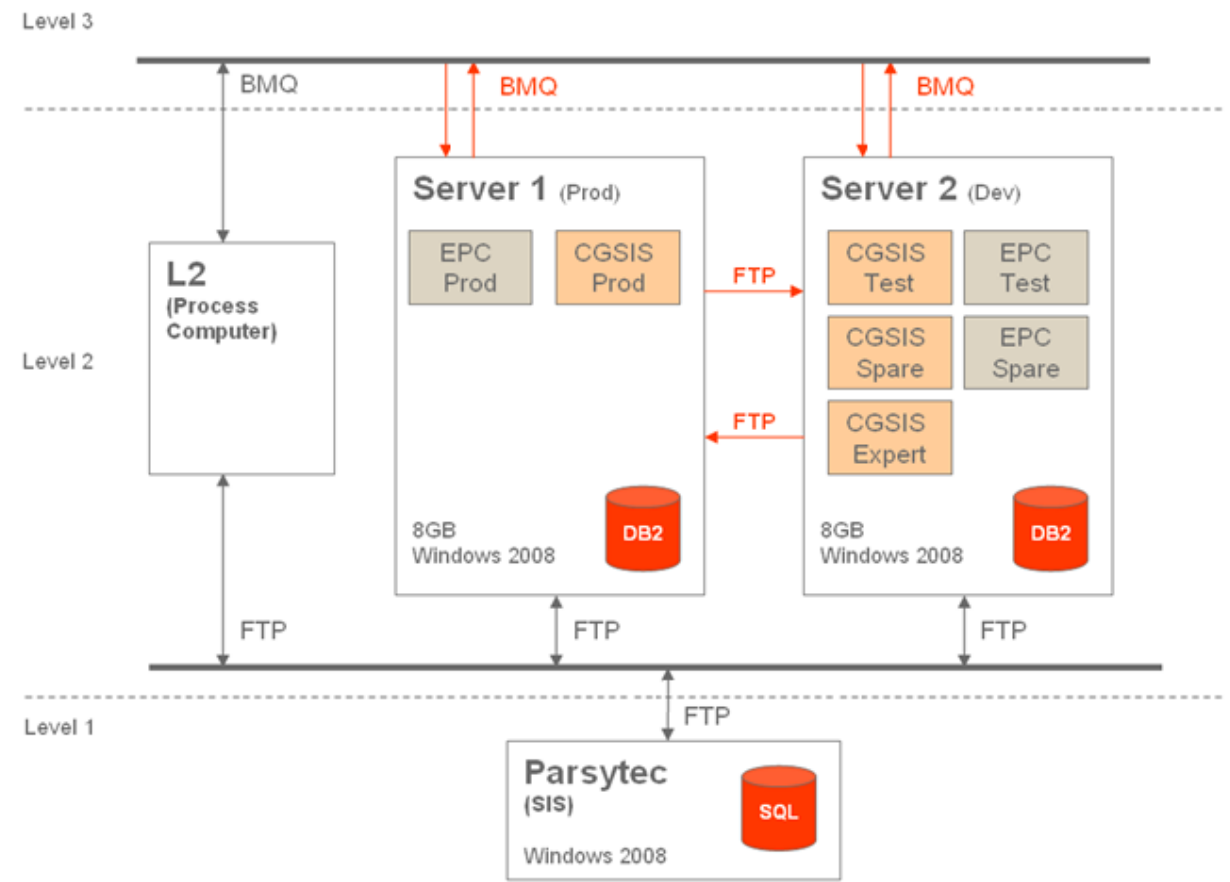

Figura 3. Interfaces de comunicação do CGSIS.

No desenvolvimento da comunicação com o nível 2 houve a necessidade de definição do código de aspecto, um parâmetro do produto que informa ao CGSIS qual a aplicação final do produto (Figura 4). Tal informação é usada para julgar se o produto atende aos critérios de qualidade para aquela aplicação. Como o CGSIS tem códigos de aspecto padronizados, foi necessário mapear no nível 2 qual código de aspecto cada produto se enquadrava, com base na informação de código de uso presente no nível 2 e que era recebido do MES.

\begin{tabular}{|l|c|l|}
\hline $\begin{array}{l}\text { Product (the status of the metal } \\
\text { as processed by the final } \\
\text { customer)(1) }\end{array}$ & Aspect code & Typical applications \\
\hline \multirow{4}{*}{ B (Black) } & 10 & Exposed parts \& specific customers \\
\cline { 2 - 3 } & 40 & $\begin{array}{l}\text { Non re-rolling critical } \\
\text { (Wheel rims (truck), gas cylinders, construction) }\end{array}$ \\
\cline { 2 - 3 } & 90 & $\begin{array}{l}\text { Standard quality } \\
\text { (+electrical steel, candelabrum, small tubes...) }\end{array}$ \\
\hline \multirow{3}{*}{ Pickled coils (P) } & 10 & Exposed parts, Specific customer, painted panels \\
\cline { 2 - 3 } & 20 & Other exposed parts (+wheel disk) \\
\cline { 2 - 3 } Cold rolled (C) & 40 & $\begin{array}{l}\text { Non re-rolling critical (tubing, wheel rims, tubing, deep } \\
\text { drawing) }\end{array}$ \\
\cline { 2 - 3 } & 80 & General products \\
\hline \multirow{2}{*}{ Hot Rolled Galvanized (H) } & 20 & Exposed \\
\hline Tin plate (T) & 70 & Not exposed \\
\cline { 2 - 3 } & 80 & Electrical steel \\
\cline { 2 - 3 } & 10 & Specific customer \\
\hline
\end{tabular}

Figura 4. Código de aspecto.

\subsection{Adequação da Operacionalização}

O sistema automatizado de inspeção de superfície de bobinas da Parsytec provê um mapa de defeitos detectados. Um técnico por turno é responsável por verificar no Parsytec todas as imagens de defeitos detectados em cada bobina produzida,

* Contribuição técnica ao $18^{\circ}$ Seminário de Automação e TI Industrial, 23 a 26 de setembro de 2014, São Paulo, SP, Brasil. 
selecionar manualmente os defeitos relevantes após verificar uma média de 500 imagens por bobina e registrar esses defeitos e seus graus de severidade no MES. Um inspetor trabalhando por turno também verifica defeitos de diferentes naturezas como forma, dimensões e processo e os registra no MES. Outro técnico trabalhando por turno analisa no MES os defeitos de cada bobina e decide se a bobina pode ser liberada ao cliente, se ela está bloqueada para envio ao cliente para análise posterior (feita por um especialista da qualidade) ou se ela deve sofrer reparo. $O$ especialista da qualidade durante o horário administrativo verifica todas as bobinas bloqueadas (cerca de $13 \%$ das bobinas produzidas) e toma a decisão final: liberação, bloqueio definitivo (cerca de $1.8 \%$ das bobinas produzidas) ou reparo. Como é possível verificar (Figura 5), existem 4 funcionários envolvidos no processo.

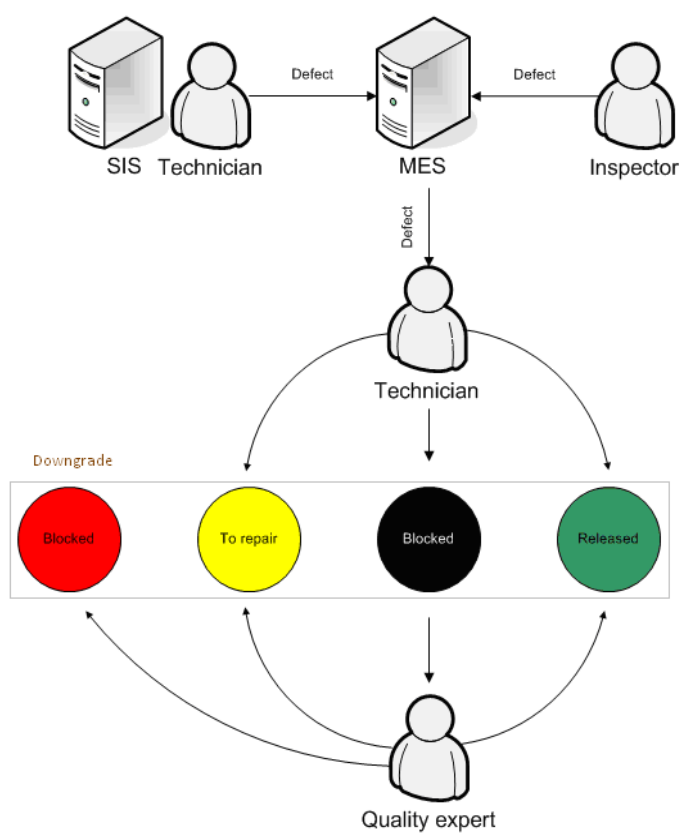

Figura 5. Processo de julgamento sem o CGSIS.

Com a implantação do CGSIS uma nova organização do processo de inspeção está prevista. Nessa nova organização, o papel do técnico responsável por verificar no SIS as imagens de todas as bobinas produzidas não existe. Defeitos detectados pelo Parsytec serão automaticamente enviados ao CGSIS. O inspetor continua a registrar defeitos de diferentes naturezas no MES e apenas um técnico por turno tomará as decisões baseadas no resultado de julgamento provido pelo CGSIS, podendo manter a indicação do CGSIS para liberação, bloqueio temporário ou reparo. O especialista da qualidade então verificará as bobinas bloqueadas temporariamente e tomará a decisão final. O CGSIS pode futuramente enviar automaticamente os resultados para o MES. Nesse caso, apenas 3 funcionários são envolvidos no processo (Figura 6). 


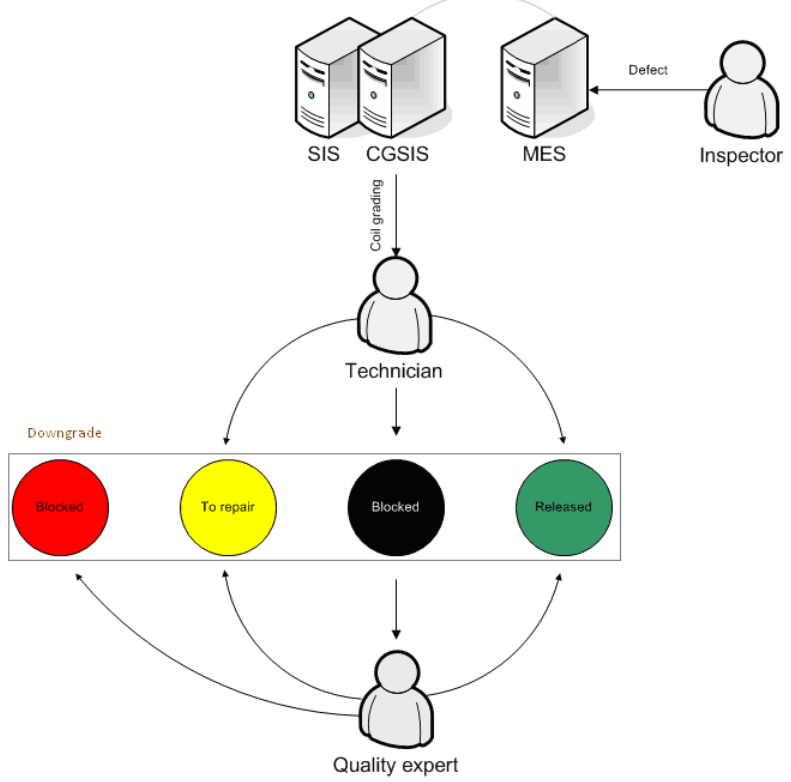

Figura 6. Processo de julgamento com o CGSIS.

\section{FUNCIONAMENTO DO SISTEMA CGSIS}

O princípio de funcionamento do CGSIS é a comparação dos cálculos efetuados sobre os dados dos defeitos recebidos do Parsytec com regras definidas pelos usuários, regras que consideram os níveis de qualidade requeridos informados pelo sistema de produção (Figura 7). As regras consideram parâmetros estatísticos dos defeitos segregados por área da bobina, tipo de defeito, intensidade e lado. Atualmente o projeto encontra-se nessa fase de definição das regras de julgamento.

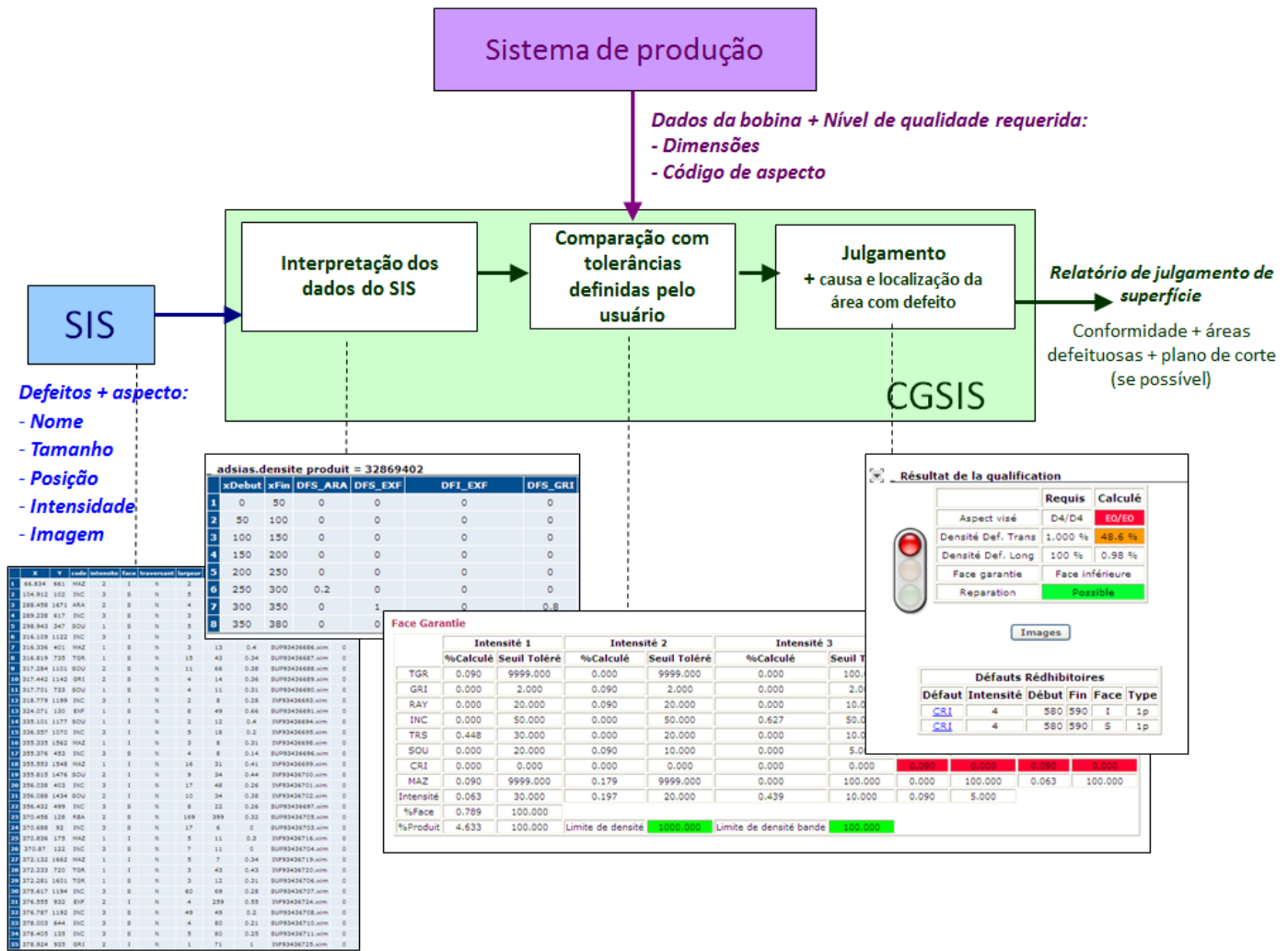

Figura 7. Processo de funcionamento do CGSIS.

* Contribuição técnica ao $18^{\circ}$ Seminário de Automação e TI Industrial, 23 a 26 de setembro de 2014, São Paulo, SP, Brasil. 


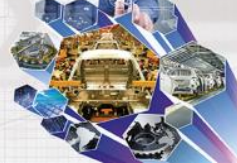

O CGSIS provê uma interface web onde é possível verificar o resultado do julgamento da qualidade de cada superfície da bobina, com o valor associado de confiança do resultado, valor que está associado à qualidade das informações de entrada do sistema, auxiliando a identificar problemas no sistema de inspeção ou interferências do ambiente (Figura 8). A interface apresenta o mapa de defeitos das faces da bobina, as anomalias identificadas e na parte superior a lista de bobinas recém produzidas e julgadas.

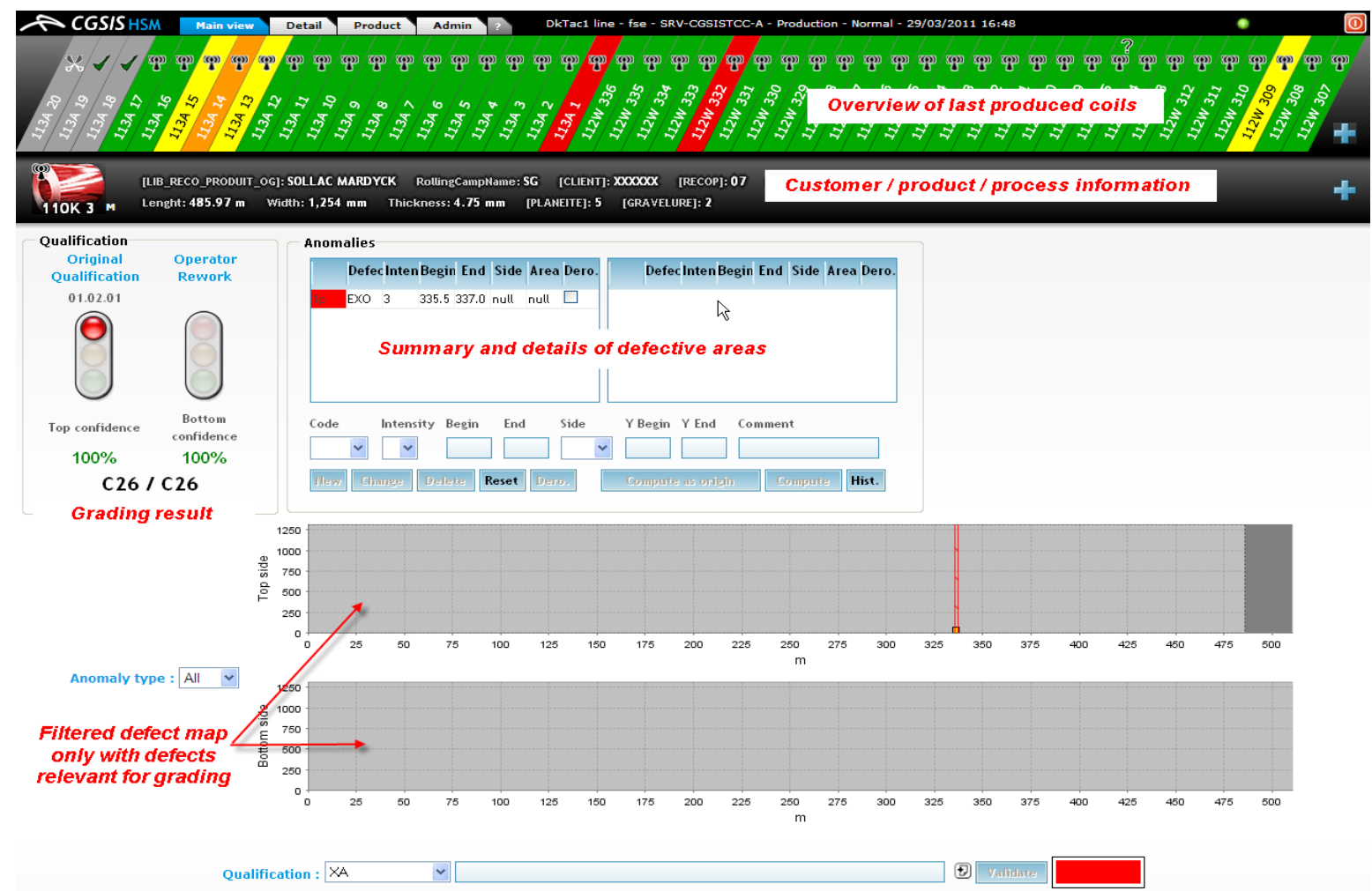

Figura 8. Interface online do CGSIS.

De acordo com a classificação do CGSIS, as bobinas são apresentadas em cores diferentes indicando sua condição em termos de qualidade superficial ou situação durante o processo (Figura 9).

\begin{tabular}{|l|l|l|l|l|l|l|}
\hline $\begin{array}{l}\text { SIS data not } \\
\text { yet received }\end{array}$ & $\begin{array}{l}\text { Coil not yet } \\
\text { graded }\end{array}$ & $\begin{array}{l}\text { Coil } \\
\text { conform }\end{array}$ & $\begin{array}{l}\text { Coil conform } \\
\text { if repaired. } \\
\text { Direct } \\
\text { cropping } \\
\text { possible }\end{array}$ & $\begin{array}{l}\text { Coil conform } \\
\text { if repaired. } \\
\text { Reparation } \\
\text { on recoiling } \\
\text { line. }\end{array}$ & $\begin{array}{l}\text { Coil not } \\
\text { conform } \\
\text { due to line } \\
\text { defects. }\end{array}$ & $\begin{array}{l}\text { Coil not conform } \\
\text { due to upstream } \\
\text { defects defects. }\end{array}$ \\
\hline \\
\hline
\end{tabular}

Figura 9. Resultado do julgamento das bobinas.

Para bobinas que apresentaram problemas, o sistema disponibiliza a imagem dos defeitos via cartografia, permitindo melhor avaliação dos defeitos encontrados e auxiliando na atuação dos inspetores de qualidade (Figura 10).

* Contribuição técnica ao $18^{\circ}$ Seminário de Automação e TI Industrial, 23 a 26 de setembro de 2014 , São Paulo, SP, Brasil. 

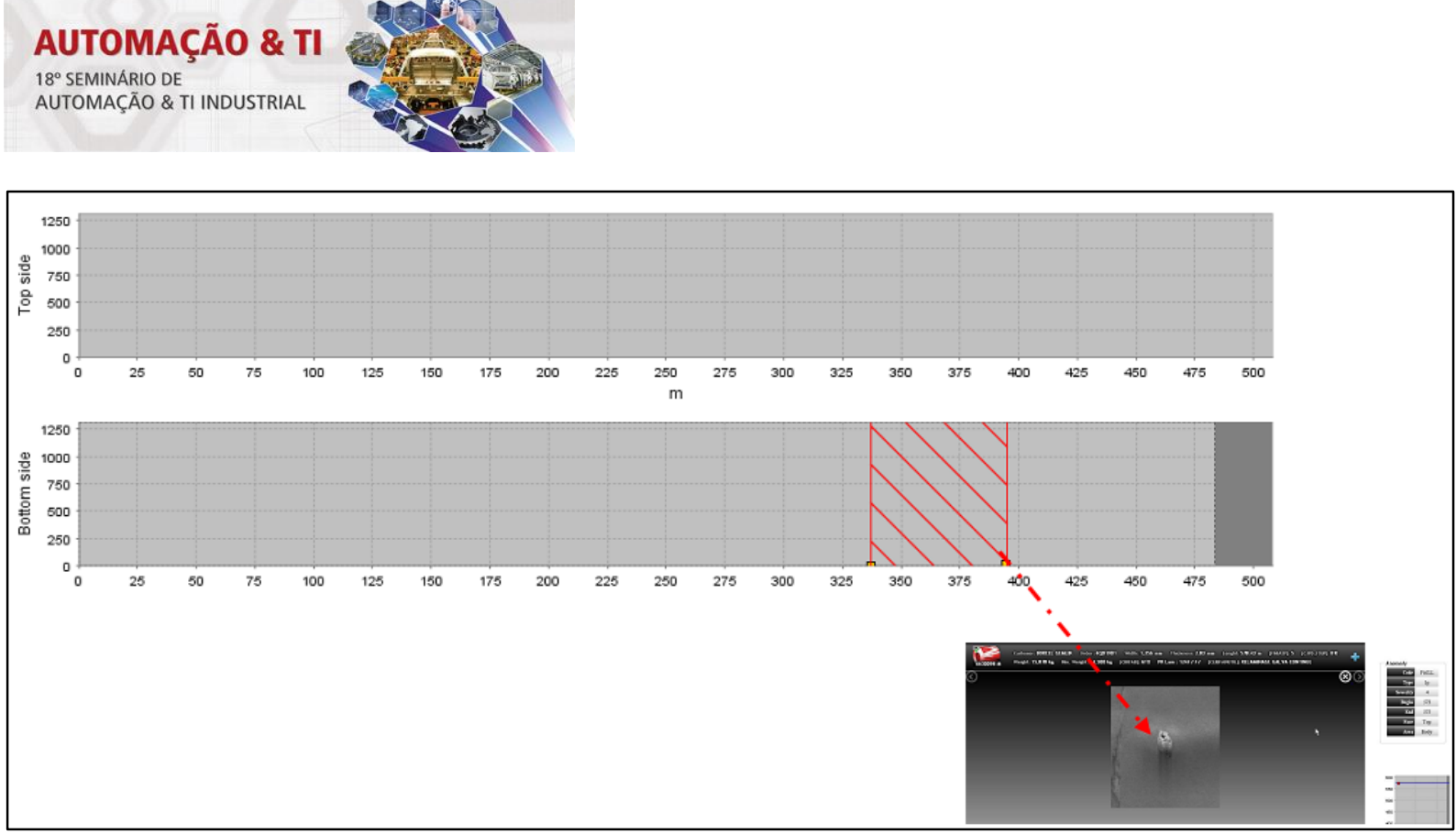

Figura 10. Cartografia de defeitos.

Atualmente o sistema encontra-se instalado na ArcelorMittal Tubarão e está em fase de testes configuração das regras de julgamento. As melhorias no sistema de inspeção para instalação do CGSIS já apresentam ganhos significativos na detecção de defeitos e sua classificação correta, melhorando a condição de julgamento não automático realizado atualmente, aumentando o aproveitamento de material e redução da reclamação de clientes. A melhoria nas condições ambientais resultou na diminuição da quantidade de imagens descartadas do sistema, reduzindo a possibilidade de julgamento inadequado. Adicionalmente, a adequação da infraestrutura para instalação do sistema possibilitou uma atualização tecnológica na arquitetura do sistema EPC, melhoria da condição de contingência e aproveitamento dos recursos existentes.

\section{CONCLUSÃO}

Inicialmente o sistema CGSIS funcionará em paralelo ao procedimento atual de inspeção até que todos os ajustes sejam realizados e seja verificada a confiabilidade dos resultados apresentados, o que se espera que ocorra no segundo semestre de 2015. Após os resultados se apresentarem confiáveis será possível reduzir o esforço e a possibilidade de falhas nos processos de inspeção e julgamento. O tempo despendido nesses processos reduzirá conforme os ajustes finos nas regras de julgamento do sistema CGSIS forem sendo realizados, o que permitirá redução de custo com pessoal na ordem de $\mathrm{R} \$ 320 \mathrm{k}$. Há ganhos também com melhoria do julgamento superficial de bobinas (repetibilidade e reprodutibilidade de resultados), evitando descartes e desclassificações desnecessárias, também no que tange o envio de bobinas para processos posteriores como decapagem e laminação a frio. Sabendo-se exatamente como é e onde está o defeito, bobinas poderão ser liberadas com certos defeitos para descartes em linhas posteriores ou para processos diferenciados evitando-se assim retrabalhos, mas estes ganhos ainda não foram avaliados/calculados.

O sistema CGSIS, como ferramenta que auxiliará no processo produtivo e controle de qualidade, demandou um trabalho de melhoria do sistema de inspeção, que resultou em um sistema atualmente mais confiável, assertivo e executando funções que antes não eram executadas. A demanda de reorganização da infraestrutura, com migração de sistemas e aproveitamento dos recursos existentes também foi

* Contribuição técnica ao $18^{\circ}$ Seminário de Automação e TI Industrial, 23 a 26 de setembro de 2014, São Paulo, SP, Brasil. 
muito demandante e resultou em uma estrutura mais atualizada e organizada, facilitando seu gerenciamento e contingência, legando um grande aprendizado para a equipe de automação. O custo total de implantação do projeto é de aproximadamente $\mathrm{R} \$ 50.000$ com equipamentos. Estiveram envolvidos diretamente no projeto um engenheiro da qualidade, três especialistas de automação, um técnico de manutenção e três especialistas do R\&D da ArcelorMittal.

Em um mercado tão competitivo, os ganhos possibilitados pela automação na forma de redução de custos, melhoria da condição de trabalho dos envolvidos e melhoria dos processos envolvidos através da garantia de resultado uniforme com base em critérios pré-estabelecidos se mostram importantes contribuições e diferencial competitivo.

\section{Agradecimentos}

Sylvain Segalini (AM R\&D França), Carlos Andrade de Almeida Pinto (AM Tubarão), Fernado José Martinelli (AM Tubarão), José Diaz Alvarez (AM R\&D Espanha), Luiz Alberto de Oliveira Martins (AM R\&D França), Claudemir Dassie (AM Tubarão), Eduardo Natal (AM Tubarão).

\section{BIBLIOGRAFIA}

1 Dalmaso R, Mendes LL, Dutra AG. Melhorias no sistema de inspeção de superfície de tiras laminadas à quente da ArcelorMittal Tubarão. In: 17 Seminário de Automação e TI Industrial, 24 a 27 de setembro de 2013, Vitória, ES, Brasil;

2 Mendes LL, Alvarez JD, Martins LAO, Dalmaso R, Guio LZ, Dutra AG. Sistema de julgamento automático de superfície para bobinas à quente - CGSIS.. In: 50ํำ Seminário de Laminação da ABM - Ouro Preto (MG) - 18 a 21 de novembro de 2013;

* Contribuição técnica ao $18^{\circ}$ Seminário de Automação e TI Industrial, 23 a 26 de setembro de 2014, São Paulo, SP, Brasil. 